\title{
Coronary Thrombus TIMI Grade Clinical Classification
}

National Cancer Institute

\section{Source}

National Cancer Institute. Coronary Thrombus TIMI Grade Clinical Classification. NCI

Thesaurus. Code C119104.

A standardized grading system developed by the Thrombolysis in Myocardial Infarction

(TIMI) Study Group to detect the presence of, and to describe the relative size of coronary thrombus via angiographic assessments. 